\title{
Efficacy of Antimicrobial Photodynamic Therapy Compared to Nystatin Therapy in reducing Candida Colony Count in Patients with Candida- Associated Denture Stomatitis: A Systematic Review and Meta-analysis
}

Parsa Firoozi ( $\sim$ parsafir2@gmail.com )

ZUMS https://orcid.org/0000-0001-5595-1485

Nima Farshidfar

School of Dentistry, Shiraz University of Medical Sciences https://orcid.org/0000-0003-2944-5305

Reza Fekrazad

AJA University of Medical Sciences

Systematic Review

Keywords: Denture stomatitis, oral candidiasis, nystatin, photodynamic antimicrobial chemotherapy, antimicrobial photodynamic therapy, Photochemotherapies

Posted Date: October 13th, 2021

DOI: https://doi.org/10.21203/rs.3.rs-689909/v1

License: (c) (i) This work is licensed under a Creative Commons Attribution 4.0 International License. Read Full License

Version of Record: A version of this preprint was published at Evidence-Based Dentistry on December 3rd, 2021. See the published version at https://doi.org/10.1038/s41432-021-0208-9. 


\section{Abstract}

Purpose: This meta-analysis assessed the efficacy of antimicrobial photodynamic therapy (aPDT) compared to conventional nystatin therapy (NYT) in reducing Candida colony count in patients with Candida-Associated Denture Stomatitis (CADS) and critically appraised the available literature.

Methods: This meta-analysis was conducted in accordance with the Preferred Reporting Items for Systematic Reviews and Meta-Analysis (PRISMA) updated guidelines. A literature search was performed in four electronic databases to identify relevant articles up to 15 August 2021 . Randomized controlled trials (RCTs) that assessed the efficacy of aPDT compared to NYT in reducing Candida colony count in patients with CADS were investigated. The weighted mean difference (MD) and 95\% confidence interval were calculated. The $P$ statistic was used to determine heterogeneity at the level of $a=0.10$. The Cochrane risk of bias (RoB 2) tool was used to assess the risk of bias. Certainty of the evidence was determined using the Grading of Recommendations Assessment, Development, and Evaluation (GRADE) ranking system.

Results: Only 3 eligible RCTs with 141 participants were included in this systematic review and meta-analysis. Based on the pooled results, NYT compared to aPDT, generally performed better in reducing Candida colony count $\left(\log _{10} \mathrm{CFU} / \mathrm{mL}\right)$ in patients' palate and patients' denture. The included studies had a moderate risk of bias and the certainty of the evidence was low.

Conclusion: Although still inconclusive, based on the current evidence aPDT may be effective in reducing Candida colony count, and treating CADS. Nonetheless, it does not appear to be more effective than conventional NYT in this regard. According to the limited number of included studies, more well-designed RCTs with larger sample sizes and standardized methodology should be conducted to validate this conclusion.

\section{Introduction}

Candida-associated denture stomatitis (CADS) is a fungal infection that presents in up to 70 percent of the edentulous denture wearers that leads to inflammation of the palatal mucosa under the denture ${ }^{1}$. Studies have raised several etiological factors for CADS, including long-term denture use, poor denture hygiene, immune system disorders, and hyposalivation ${ }^{2}$. In addition, it has been suggested that colonization of opportunistic Candida species, especially Candida albicans, play an important role in the pathogenesis of the disease ${ }^{3-5}$.

For the treatment of CADS, denture hygiene should be strengthened, proper fitness of the denture should be evaluated, and the age of denture should be taken into account ${ }^{6}$. Additionally, patients can prevent CADS by using chlorhexidine mouthwashes, soaking denture in water, disinfecting denture with appropriate disinfectants, and removing denture during sleep. Dentists, on the other hand, can prevent CADS by reviewing their patients' dentures and replacing/relining the dentures if required ${ }^{7}$.

Nystatin, Miconazole, and the other topical antifungals are the most common agents prescribed for the treatment of CADS and in cases with recurrent and severe CADS, systemic antifungal agents are prescribed which are associated with severe conditions such as hepatic necrosis, allergic reactions, adrenal insufficiency, and drug interactions $4,8-10$. Other problems are associated with the continuous use of antifungal drugs including the resistance of fungal species and recurrence of CADS after treatment ${ }^{11-13}$. Therefore, it is necessary to seek safe treatments with fewer side effects. Antimicrobial photodynamic therapy (aPDT) is based on the interaction of three components including light, photosensitizer (PS), and oxygen ${ }^{14}$. In aPDT process, light sources can be selected from non-coherent (Light Emitting Diode (LED)), coherent (laser), or even broadband spectrum (lamp) light sources ${ }^{15}$. Methylene blue (MB), toluidine blue ortho (TBO), and indocyanine green (ICG) are the most common PSs used in dentistry ${ }^{16,17}$. PS absorbs energy from a light source and alters from the ground state to the excited triplet state. More specifically, the PS in the excited triplet state then either loses energy and returns to the ground state or is put in the direction of one of two types of reactions, Type I and Type II ${ }^{18}$.

The Type I reaction occurs when energy is transferred to hydrogen or an electron. This energy transfer induces the production of free radicals in nearby molecules, which produces reactive oxygen species (ROS) as they react with oxygen ${ }^{19}$. Type II reactions are those in which PS excited energy is directly transferred to the ${ }^{3} \mathrm{O}_{2}$ oxygen molecule and resulting in the formation of ${ }^{1} \mathrm{O}_{2}$ or ROS ${ }^{18}$. Accordingly, microorganisms such as Candida species are destroyed through this oxidative stress ${ }^{20}$.

Hamblin et al. in $2020^{21}$ proposed an oxygen-independent antimicrobial photoinactivation as a type III photochemical mechanism in aPDT. In this procedure, specific PSs, such as psoralens and tetracyclines, could bind to a specific molecular structure within a target microorganism and then be activated with short-wavelength light (i.e., UVA or blue) without the need for oxygen. Microorganisms are eliminated as a result of the formation of covalent adducts in their DNA or ribosomes ${ }^{21}$.

It has been demonstrated that antimicrobial photodynamic therapy (aPDT) can be used to treat periodontal diseases, peri-implant diseases, lichen planus, and a variety of fungal infections ${ }^{22-25}$. An in vitro study showed that aPDT can be an effective method for reducing Candida species on resin acrylic dentures ${ }^{26}$. Also, a few randomized controlled trials (RCTs) have recently been published which have suggested aPDT as a promising solution for CADS treatment $20,27,28$. 
The objective of the present systematic review and meta-analysis was to compare the efficacy of aPDT with nystatin therapy (NYT) in reducing Candida colony count in patients with CADS and critically appraise the available literature to reveal research gaps in this regard.

\section{Materials And Methods}

The protocol of this systematic review and meta-analysis was previously registered in PROSPERO (CRD42021243146). The meta-analysis was elaborated in accordance with the Preferred Reporting Items for Systematic Reviews and Meta-Analysis (PRISMA) updated guideline ${ }^{29}$. In this study, recently published systematic review models were adopted ${ }^{30,31}$.

\subsection{Eligibility criteria}

Studies were included based on the PICOS components:

Participants $(P)$ : Edentulous individuals diagnosed with CADS.

Intervention (I): aPDT with any types of light sources and PS agents.

Comparator (C): Conventional NYT.

Outcome (0): The primary outcome included reduction of Candida colony count $\left(\log _{10} \mathrm{CFU} / \mathrm{mL}\right)$ in patients' palate and denture.

Study type (S): RCTs

Studies were excluded if they:

1. Had not reported Candida colony count values.

2. Had included both NYT and aPDT in the same treatment group.

\subsection{Information sources and search strategy}

Before 15 August 2021, a comprehensive literature search was conducted by two independent authors (PF, NF) in MEDLINE (PubMed), Cochrane Central Register of Controlled Trials (CENTRAL), Scopus, and Web of Science electronic databases. Also, Google Scholar was searched as an adjunctive tool to find additional eligible studies. The first 100 relevant records were evaluated from this "grey literature" search and considered for inclusion.

Any record relevant to RCTs comparing aPDT and NYT in terms of CADS treatment included for further screening with no restrictions regarding the language. Also, a manual search of the reference lists of relevant articles was conducted. Searches were limited to published and peer-reviewed studies from up to August 2021. A literature search of the aforementioned databases was conducted using MeSH terms and relevant free keywords (Supplementary file 1).

Study selection

All records were imported into the EndNote software (version 9.3), and duplicate records were removed. Then titles and abstracts of all retrieved records were prescreened for potentially eligible studies. The full-text version of the previously identified studies was obtained and then evaluated in detail with scrutiny according to predetermined eligibility criteria for inclusion in the review. Two reviewers (PF, NF) performed the screening process and study selection independently. Any Disagreement about eligibility and any controversies between the two reviewers were resolved through a discussion with the third reviewer (RF) until a consensus was reached. Kappa test was used to assess the inter-reviewer reliability (0.8 as an acceptable threshold value).

\subsection{Data collection and data items}

The following data were obtained from included studies: Authors, year of publication, study design, sample size, Candida species, light source characteristics, pre-irradiation time for PS agent, type of PS agent, antifungal agent characteristics, and follow-up period. For the meta-analysis, the means and standard deviations regarding the Candida colony count values and overall clinical success rate were extracted. Two reviewers (PF, RF) performed the data collection process independently. An Excel predetermined table was used to collect data electronically.

\subsection{Risk of bias assessment}

Two authors (PF, NF) independently assessed the quality and the risk of bias in this review. Risk of bias was assessed using the Cochrane Risk of Bias (RoB 2) tool $^{32}$ for randomized controlled trials which addresses five main domains: randomization process, deviations from intended interventions, missing outcome data, measurement of the outcome, and selection of the reported result.

\subsection{Synthesis methods}

Page $3 / 11$ 
All statistical analysis was performed using Review Manager (RevMan, Version 5.4.1, Cochrane Community). The primary outcome included the reduction of Candida colony count in patients' palate and denture. Accordingly, all different units of Candida colony count values were transformed into $\log _{10} \mathrm{CFU} / \mathrm{mL}$ then analysis was performed on $\mathrm{Log}_{10} \mathrm{CFU} / \mathrm{mL}$ to compare mycological efficacy of aPDT with NYT in the treatment of CADS. The meta-analysis was performed by measuring the means difference (MD) for continuous variables. Ninety-five percent confidence interval (CI) was used to present all the outcomes, and $P<0.05$ was considered statistically significant. The $P^{2}$ statistic was used to determine heterogeneity at the level of $\mathrm{a}=0.10$. The fixed-effect model was used when $I^{2}=0 \%$ and the random effect model was applied in cases with $l^{2}>50 \%$. Sensitivity analysis was not performed and funnel plots were not constructed due to a few numbers of included RCTs ${ }^{33}$.

\subsection{Certainty assessment}

The certainty of the evidence was assessed using the Grading of Recommendations Assessment, Development, and Evaluation (GRADE) ranking system through five analysis criteria (risk of bias, inconsistency, indirect evidence, imprecision, and publication bias). Thus, the quality of evidence was classified as high, moderate, low, or very low certainty of the evidence.

\section{Results}

\subsection{Study selection}

In total, the initial search strategies yielded 46 articles. Of the initial searches, 7 studies were retained for further investigation and 3 articles were excluded primarily based on their lack of appropriate endpoints matching our search criteria ${ }^{34-36}$. Finally, 4 randomized controlled trials were initially identified carefully from the proposed electronic databases, of which one study was omitted due to dual intervention (aPDT + NYT) in comparison with NYT ${ }^{37}$. Hence, based on the eligibility criteria, 3 RCTs were considered for qualitative and quantitative analysis $20,27,28$ (Fig. 1). The interrater agreement in terms of study selection (kappa) was 0.95 .

\subsection{Study characteristics}

One hundred forty-one participants with CADS were included in this systematic review and meta-analysis. The mean age of the participants varied from 61.25 to 70. All included studies proposed Candida albicans, Candida tropicalis, and Candida glabrata as the most prevalent Candida species responsible for CADS. All studies used the oral suspension form of nystatin $(100.000 \mathrm{Ul} / \mathrm{mL})$ as an antifungal agent in comparison with aPDT in the treatment of CADS. Moreover, two studies used LED ${ }^{20,27}$ and one study ${ }^{28}$ used the diode laser as the light source. Light source wavelength ranged from $455 \mathrm{~nm}$ to $660 \mathrm{~nm}$. Pre-irradiation period and treatment interval varied from $10 \mathrm{~min}$ to $30 \mathrm{~min}$ and $4 \mathrm{~min}$ to $26 \mathrm{~min}$, respectively. The main characteristics of the included studies are briefly illustrated in Table 1.

\subsection{Results of included studies}

Mima et al. compared the efficacies of aPDT to NYT ( $n=20$ each) for the treatment of CADS. Mycological cultures were taken from the denture and palate at baseline (day 0), at the end of the treatments (day 15), and at the follow-up time intervals (days 30,60 , and 90 ). It was assessed that at days 15 and 30, Candida colony count was significantly reduced in patients' palate and denture for both NYT and aPDT groups, compared to the baseline (day 0$)(P<0.05)$. Candida colony count on day 60 of follow-up intervals showed a significant increase compared to obtained values at the end of treatments $(P<0.05)$. Additionally, no statistically significant difference was shown between the Candida colony count values obtained from the aPDT and the NYT groups in all time intervals. Therefore, it was concluded that aPDT would be as effective as NYT in reducing Candida colony count $^{20}$.

In another recent study by Alrabiah et al., Candida colony count was measured from denture and palate at baseline (day 0 ), at the end of the treatments (day 15), and on the follow-up time intervals (days 30, and 60). A substantial decrease was observed in the Candida colony count mean values from denture and palate at the end of the treatments (day 15) compared to the baseline (day 0 ) in both treatment groups. On follow-up time intervals (days 30 and 60) a significant reduction in Candida colony count was observed in the NYT group only $(P<0.05)$. The aforementioned reduction was significantly higher on day 15 compared to follow-up days. However, there was no statistically significant difference between aPDT and NYT regarding the Candida colony count mean values obtained in all time intervals. Hence, aPDT was concluded as equally effective as NYT in reducing Candida colony count ${ }^{28}$.

Similar to the previous studies, Alves et al. evaluated the efficacy of aPDT ( $n=30)$ in comparison with NYT $(n=35)$ for the treatment of CADS. The efficacy of the treatments was verified by Candida colony count values from denture and palate at baseline (day 0 ), at the end of the treatments (day 15), and on the follow-up time intervals (days 30,45, and 60). A significant decline in Candida colony count was shown at the end of the treatments compared to the baseline $(P=0.018)$. The authors showed that aPDT can be a promising treatment for CADS and had a comparable result to NYT in terms of reduction in Candida colony count.

\subsection{Quality assessment}

All included studies had an overall moderate risk of bias. The randomization process was not described appropriately in one study ${ }^{20}$. Additionally, some concerns were observed regarding the deviations from the intended interventions in all included studies ${ }^{20,27,28}$. None of the included studies 
had missing outcome data. One study had a moderate risk of bias in terms of measurement of the outcome ${ }^{28}$. Regarding the selection of the reported result, all studies had a moderate to high risk of bias $20,27,28$ (Fig. 2).

\subsection{Meta-analysis}

Three RCTs were selected for meta-analysis $20,27,28$. Mycological comparisons were conducted on the Candida colony count ( $\left.\log _{10} C F U / m L\right)$ in patients' palate and denture at the end of the treatments (day 15) and on follow-up intervals (days 30, and 60).

\subsubsection{Mycological efficacy of aPDT compared to NYT in palate}

At the end of the treatments (day 15) NYT was superior to aPDT in reducing Candida colony count $\left(\log _{10} C F U / m L\right)$ in patients' palate $(\mathrm{MD}=0.58$; $95 \% \mathrm{Cl}[0.24,0.92] ; P=0.0008 ; P=0 \%)$.

Similarly, on day 30, NYT compared to aPDT more reduced Candida colony count (MD $\left.=0.60 ; 95 \% \mathrm{Cl}[0.18,1.02] ; P=0.005 ; P^{2}=0 \%\right)$ in patients' palate.

Nonetheless, on day 60 of follow-up period no statistically significant difference was shown between aPDT and NYT (MD = 0.24; $95 \% \mathrm{Cl}[-0.23,0.70]$; $P=0.31 ; P=0 \%)$ (Fig. 3).

\subsubsection{Mycological efficacy of aPDT compared to NYT in denture}

At the end of the treatments (day 15) NYT was superior to aPDT in reducing Candida colony count $\left(\log _{10} C F U / m L\right)$ in patients' denture $(\mathrm{MD}=1.26$; $\left.95 \% \mathrm{Cl}[0.36,2.16] ; P=0.006 ; P^{2}=0 \%\right)$. However, on day $30(\mathrm{MD}=2.59 ; 95 \% \mathrm{Cl}[-0.55,5.73] ; P=0.11 ; P=63 \%)$ and day $60(\mathrm{MD}=0.94 ; 95 \% \mathrm{Cl}[-0.11$, 1.99]; $P=0.08 ; P=0 \%)$ of follow-up period no statistically significant difference was shown between aPDT and NYT (Fig. 4).

\subsection{Certainty of evidence}

Based on the GRADE scale, the certainty of evidence in terms of reducing Candida colony count with aPDT compared to NYT is low (Table 2). The moderate risk of bias and small sample size of the included studies would introduce a serious concern for the analysis.

\section{Discussion}

Some RCTs have evaluated the efficacy of aPDT in treating CADS and compared it with NYT 20,27, 28 . Despite finding only three RCTs meeting our inclusion criteria, we were able to conduct a meta-analysis to compare aPDT with NYT in reducing Candida colony count which was not conducted in the previously published qualitative systematic reviews ${ }^{31,38}$. Moreover, the previous systematic review has not assessed the risk of bias in included studies ${ }^{38}$. Therefore, the current meta-analysis attempted to solve the shortcomings of the previous systematic reviews and provide an evidence-based conclusion based on the available literature.

Even though some in vitro and animal studies ${ }^{39-42}$ have been evaluated aPDT on oral candidiasis, since the oral environment of humans is different from animals, and factors such as biofilm and microbiota composition, salivary flux, oral hygiene, and food habits might change the response to aPDT, it is difficult to compare the findings of RCTs with those of in-vitro and animal studies ${ }^{20}$.

Qualitative results of all included RCTs showed that aPDT is effective in reducing Candida colony count, and thus in improving CADS. Additionally, RCTs did not show a statistically significant difference between aPDT and NYT in reducing Candida colony count in all time intervals (days 15,30 , $45,60$, and 90$)^{20,27,28}$ which was in line with the conclusion of the previously published systematic review ${ }^{31}$.

Qualitative results of this systematic review support the results of each included RCT and previously published systematic reviews ${ }^{31,38}$ in terms of the efficacy of aPDT in reducing Candida colony count. Nevertheless, the meta-analysis results showed that NYT statistically is superior to aPDT in reducing Candida colony count at the end of the treatments (day 15) in both patients' palate and denture. Although the results of each included study showed that there was no statistically significant difference between aPDT and NYT in reducing Candida colony count in all time intervals, it seems that the small sample size of these included studies caused the contradiction between their results and the pooled results of the performed meta-analysis.

On day 30, a statistically significant difference was observed between aPDT and NYT groups in reducing Candida colony count in patients' palate, however, this difference was not significant in patients' denture. This may be justified by the porous and uneven inner surface of acrylic resin dentures which act as a reservoir for microorganisms that leads for faster recolonization in patients' denture compared to patients' palate after treatments.

Also, the lack of significant difference between aPDT and NYT on day 60 may be justified by the inner surface of acrylic resin dentures which act as a reservoir for Candida species ${ }^{43}$, and their tendency to recolonize on hard surfaces such as patients' denture and palate immediately after treatments. The aforementioned recolonization may lead to the recurrence of CADS after therapeutic sessions.

Page 5/11 
Additionally, based on the meta-analysis more success in the NYT group in reducing Candida colony count on the aforementioned intervals is probably because NYT can eliminate Candida species in other areas of the mouth, such as the buccal mucosa and tongue; however, utilized aPDT technique among studies only affected the palatal mucosa $20,27,28$. It seems that if aPDT is not limited to the palate of patients and is used in the whole oral cavity, the same results may be obtained compared to NYT. In addition, the size of fungal cells compared to bacterial cells seems to affect the success of aPDT in reducing Candida colony count, because larger cells need more amount of singlet oxygen to be killed ${ }^{44}$. Besides, according to the meta-analysis, the lower success in reducing Candida colony count in the aPDT group can be justified by the fact that the number of aPDT sessions was less than that of NYT. On this basis, patients get aPDT only in the office; however, patients in the NYT group used nystatin several times a day $20,27,28$. It is possible to achieve the same results compared to NYT by designing a low-cost aPDT devices for home use as is mentioned in a study 45 .

It also seems that the parameters related to light sources and types of PSs in the aPDT group are associated with the reduction in Candida colony count in aPDT group ${ }^{27}$. Even though the research designs of the included studies were the same, nonetheless, the methodologies used in aPDT varied significantly. One of the most important barriers was a lack of standardization of light source and PS parameters across trials. While conducting the meta-analysis, aPDT was utilized as a catch-all term for any aPDT setting (independent of parameters).

The current systematic review and meta-analysis has shown the promising effect of aPDT in reducing Candida colony count ( $\left.\mathrm{Log}_{10} \mathrm{CFU} / \mathrm{mL}\right)$ and this effect does not seem to be as effective as NYT. Moreover, the clinical significance of these results must be carefully weighed (Although statistically significant, the clinical significance should be questioned).

This meta-analysis had some limitations that must be considered and due to these limitations, the results should be interpreted with caution. The first limitation was the lack of an appropriate number of RCTs for inclusion. Another limitation was associated with the moderate quality of the included studies. Moreover, the small sample size was the obvious shortcoming of each included study which probably led to inconsistent results between the meta-analysis and the results of each included RCT in terms of comparing aPDT and NYT in reducing Candida colony count. Furthermore, Finding the most effective treatment parameters of aPDT has not yet been established in this review.

\section{Conclusions}

Within the limitations of the current systematic review and meta-analysis, it can be concluded that aPDT may be beneficial in reducing the Candida colony count in patients' palate and denture, however, it does not seem to be effective as NYT in this regard. Due to the limited number of included RCTs and the moderate quality of these studies, more well-designed RCTs with larger sample sizes are recommended to compare aPDT with NYT in treatment of CADS.

\section{Declarations}

\section{Ethics declarations}

The authors declare no conflicts of interest.

\section{Author contributions}

PF: Study selection, data extraction, statistical analysis, quality appraisal, wrote manuscript

NF: Study selection, data extraction quality appraisal, edited manuscript

RF: Project design, study selection, final revision

\section{References}

1. Barbeau J, Séguin J, Goulet JP, de Koninck L, Avon SL, Lalonde B, et al. (2003) Reassessing the presence of Candida albicans in denture-related stomatitis. Oral Surg Oral Med Oral Pathol Oral Radiol Endod 95(1):51-9.

2. Figueiral MH, Azul A, Pinto E, Fonseca PA, Branco FM, Scully C (2007) Denture-related stomatitis: identification of aetiological and predisposing factors - a large cohort. J Oral Rehabil 34(6):448-55.

3. Lavaee F, Motaghi D, Jassbi AR, Jafarian H, Ghasemi F, Badiee P (2018) Antifungal effect of the bark and root extracts of Punica granatum on oral Candida isolates. Curr Med Mycol 4(4):20-4.

4. Chandra J, Mukherjee PK, Leidich SD, Faddoul FF, Hoyer LL, Douglas LJ, et al. (2001) Antifungal resistance of candidal biofilms formed on denture acrylic in vitro. J Dent Res 80(3):903-8.

5. Jeganathan S, Lin CC (1992) Denture stomatitis-a review of the aetiology, diagnosis and management. Aust Dent J 37(2):107-14.

6. Pappas PG, Kauffman CA, Andes D, Benjamin DK, Jr., Calandra TF, Edwards JE, Jr., et al. (2009) Clinical Practice Guidelines for the Management Candidiasis: 2009 Update by the Infectious Diseases Society of America. Clin Infect Dis 48(5):503-35.

Page 6/11 
7. Hannah VE, O'Donnell L, Robertson D, Ramage G (2017) Denture Stomatitis: Causes, Cures and Prevention. Prim Dent J 6(4):46-51.

8. Yarborough A, Cooper L, Duqum I, Mendonça G, McGraw K, Stoner L (2016) Evidence Regarding the Treatment of Denture Stomatitis. J Prosthodont 25(4):288-301.

9. Ghapanchi J, Lavaee F, Moattari A, Shakib M (2015) The antibacterial effect of four mouthwashes against streptococcus mutans and escherichia coli. J Pak Med Assoc 65(4):350-3.

10. Sanglard D, Coste A, Ferrari S (2009) Antifungal drug resistance mechanisms in fungal pathogens from the perspective of transcriptional gene regulation. FEMS Yeast Res 9(7):1029-50.

11. Shapiro RS, Robbins N, Cowen LE (2011) Regulatory circuitry governing fungal development, drug resistance, and disease. Microbiol Mol Biol Rev 75(2):213-67.

12. Perea S, Patterson TF (2002) Antifungal resistance in pathogenic fungi. Clin Infect Dis 35(9):1073-80.

13. PALLASCH TJ (2002) Antifungal and antiviral chemotherapy. Periodontology 2000 28(1):240-55.

14. Azaripour A, Dittrich S, Van Noorden CJF, Willershausen B (2018) Efficacy of photodynamic therapy as adjunct treatment of chronic periodontitis: a systematic review and meta-analysis. Lasers Med Sci 33(2):407-23.

15. Brancaleon L, Moseley H (2002) Laser and non-laser light sources for photodynamic therapy. Lasers Med Sci 17(3):173-86.

16. Gomer CJ (1991) Preclinical examination of first and second generation photosensitizers used in photodynamic therapy. Photochem Photobiol 54(6):1093-107.

17. Fekrazad R, Khoei F, Bahador A, Hakimiha N (2020) Comparison of different modes of photo-activated disinfection against Porphyromonas gingivalis: an in vitro study. Photodiagnosis Photodyn Ther:101951.

18. de Oliveira AB, Ferrisse TM, Marques RS, de Annunzio SR, Brighenti FL, Fontana CR (2019) Effect of photodynamic therapy on microorganisms responsible for dental caries: A systematic review and meta-analysis. International journal of molecular sciences 20(14):3585.

19. Reis ACM, Regis WFM, Rodrigues LKA (2019) Scientific evidence in antimicrobial photodynamic therapy: an alternative approach for reducing cariogenic bacteria. Photodiagnosis and Photodynamic Therapy 26:179-89.

20. Mima EG, Vergani CE, Machado AL, Massucato EM, Colombo AL, Bagnato VS, et al. (2012) Comparison of Photodynamic Therapy versus conventional antifungal therapy for the treatment of denture stomatitis: a randomized clinical trial. Clin Microbiol Infect 18(10):E380-8.

21. Hamblin MR, Abrahamse H (2020) Oxygen-Independent Antimicrobial Photoinactivation: Type III Photochemical Mechanism? Antibiotics $9(2): 53$.

22. Akram Z, Al-Shareef SA, Daood U, Asiri FY, Shah AH, AlQahtani MA, et al. (2016) Bactericidal Efficacy of Photodynamic Therapy Against Periodontal Pathogens in Periodontal Disease: A Systematic Review. Photomed Laser Surg 34(4):137-49.

23. Akram Z, Hyder T, Al-Hamoudi N, Binshabaib MS, Alharthi SS, Hanif A (2017) Efficacy of photodynamic therapy versus antibiotics as an adjunct to scaling and root planing in the treatment of periodontitis: A systematic review and meta-analysis. Photodiagnosis and Photodynamic Therapy 19:86-92.

24. Zeza B, Farina R, Pilloni A, Mongardini C (2018) Clinical outcomes of experimental gingivitis and peri-implant mucositis treatment with professionally administered plaque removal and photodynamic therapy. Int J Dent Hyg 16(2):e58-e64.

25. Akram Z, Javed F, Hosein M, Al-Qahtani MA, Alshehri F, Alzahrani Al, et al. (2018) Photodynamic therapy in the treatment of symptomatic oral lichen planus: A systematic review. Photodermatol Photoimmunol Photomed 34(3):167-74.

26. Mima EG, Pavarina AC, Ribeiro DG, Dovigo LN, Vergani CE, Bagnato VS (2011) Effectiveness of photodynamic therapy for the inactivation of Candida spp. on dentures: in vitro study. Photomed Laser Surg 29(12):827-33.

27. Alves F, Carmello JC, Alonso GC, Mima EGdO, Bagnato VS, Pavarina AC (2020) A randomized clinical trial evaluating Photodithazine-mediated Antimicrobial Photodynamic Therapy as a treatment for Denture stomatitis. Photodiagnosis and Photodynamic Therapy 32:102041.

28. Alrabiah M, Alsahhaf A, Alofi RS, Al-Aali KA, Abduljabbar T, Vohra F (2019) Efficacy of photodynamic therapy versus local nystatin in the treatment of denture stomatitis: A randomized clinical study. Photodiagnosis and Photodynamic Therapy 28:98-101.

29. Page MJ, McKenzie JE, Bossuyt PM, Boutron I, Hoffmann TC, Mulrow CD, et al. (2021) The PRISMA 2020 statement: an updated guideline for reporting systematic reviews. BMJ 372:n71.

30. da Costa RMB, Poluha RL, De la Torre Canales G, Junior JFS, Conti PCR, Neppelenbroek KH, et al. (2020) The effectiveness of microwave disinfection in treating Candida-associated denture stomatitis: a systematic review and metaanalysis. Clinical Oral Investigations 24(11):382132.

31. Davoudi A, Ebadian B, Nosouhian S (2018) Role of laser or photodynamic therapy in treatment of denture stomatitis: A systematic review. J Prosthet Dent 120(4):498-505.

32. Sterne JAC, Savović J, Page MJ, Elbers RG, Blencowe NS, Boutron I, et al. (2019) RoB 2: a revised tool for assessing risk of bias in randomised trials. BMJ 366:14898.

33. Deeks JJ, Higgins JP, Altman DG, Group obotCSM. Analysing data and undertaking meta-analyses. Cochrane Handbook for Systematic Reviews of Interventions2019. p. 241-84.

Page $7 / 11$ 
34. de Senna AM, Vieira MMF, Machado-de-Sena RM, Bertolin AO, Núñez SC, Ribeiro MS (2018) Photodynamic inactivation of Candida ssp. on denture stomatitis. A clinical trial involving palatal mucosa and prosthesis disinfection. Photodiagnosis Photodyn Ther 22:212-6.

35. Labban N, Taweel SMA, MA AL, Alfouzan AF, Alshiddi IF, Assery MK (2021) Efficacy of Rose Bengal and Curcumin mediated photodynamic therapy for the treatment of denture stomatitis in patients with habitual cigarette smoking: A randomized controlled clinical trial. Photodiagnosis Photodyn Ther 35:102380.

36. Maciel CM, Piva MR, Ribeiro MA, de Santana Santos T, Ribeiro CF, Martins-Filho PR (2016) Methylene Blue-Mediated Photodynamic Inactivation Followed by Low-Laser Therapy versus Miconazole Gel in the Treatment of Denture Stomatitis. J Prosthodont 25(1):28-32.

37. Afroozi B, Zomorodian K, Lavaee F, Zare Shahrabadi Z, Mardani M (2019) Comparison of the efficacy of indocyanine green-mediated photodynamic therapy and nystatin therapy in treatment of denture stomatitis. Photodiagnosis and Photodynamic Therapy 27:193-7.

38. Javed F, Samaranayake LP, Romanos GE (2014) Treatment of oral fungal infections using antimicrobial photodynamic therapy: a systematic review of currently available evidence. Photochem Photobiol Sci 13(5):726-34.

39. Donnelly RF, McCarron PA, Tunney MM (2008) Antifungal photodynamic therapy. Microbiol Res 163(1):1-12.

40. Konopka K, Goslinski T (2007) Photodynamic therapy in dentistry. J Dent Res 86(8):694-707.

41. Mima EG, Pavarina AC, Dovigo LN, Vergani CE, Costa CA, Kurachi C, et al. (2010) Susceptibility of Candida albicans to photodynamic therapy in a murine model of oral candidosis. Oral Surg Oral Med Oral Pathol Oral Radiol Endod 109(3):392-401.

42. Trindade FZ, Pavarina AC, Ribeiro AP, Bagnato VS, Vergani CE, Costa CA (2012) Toxicity of photodynamic therapy with LED associated to Photogem®: an in vivo study. Lasers Med Sci 27(2):403-11.

43. Salerno C, Pascale M, Contaldo M, Esposito V, Busciolano M, Milillo L, et al. (2011) Candida-associated denture stomatitis. Med Oral Patol Oral Cir Bucal 16(2):e139-43.

44. Stranadko EP, Ponomarev GV, Mechkov V, Ryabov M, Ivanov AV, Reshetnickov AV, et al., editors. First experience of photodithazine clinical application for photodynamic therapy of malignant tumors. Optical Methods for Tumor Treatment and Detection: Mechanisms and Techniques in Photodynamic Therapy IX; 2000: International Society for Optics and Photonics.

45. Momchilov N, Bliznakova I, Borisova E, Troyanova P, Avramov L (2007) Development of Low-Cost Photodynamic Therapy Device. Acta Physica Polonica Series a 112726419.

\section{Tables}

Table 1 Main characteristics of the included studies. 


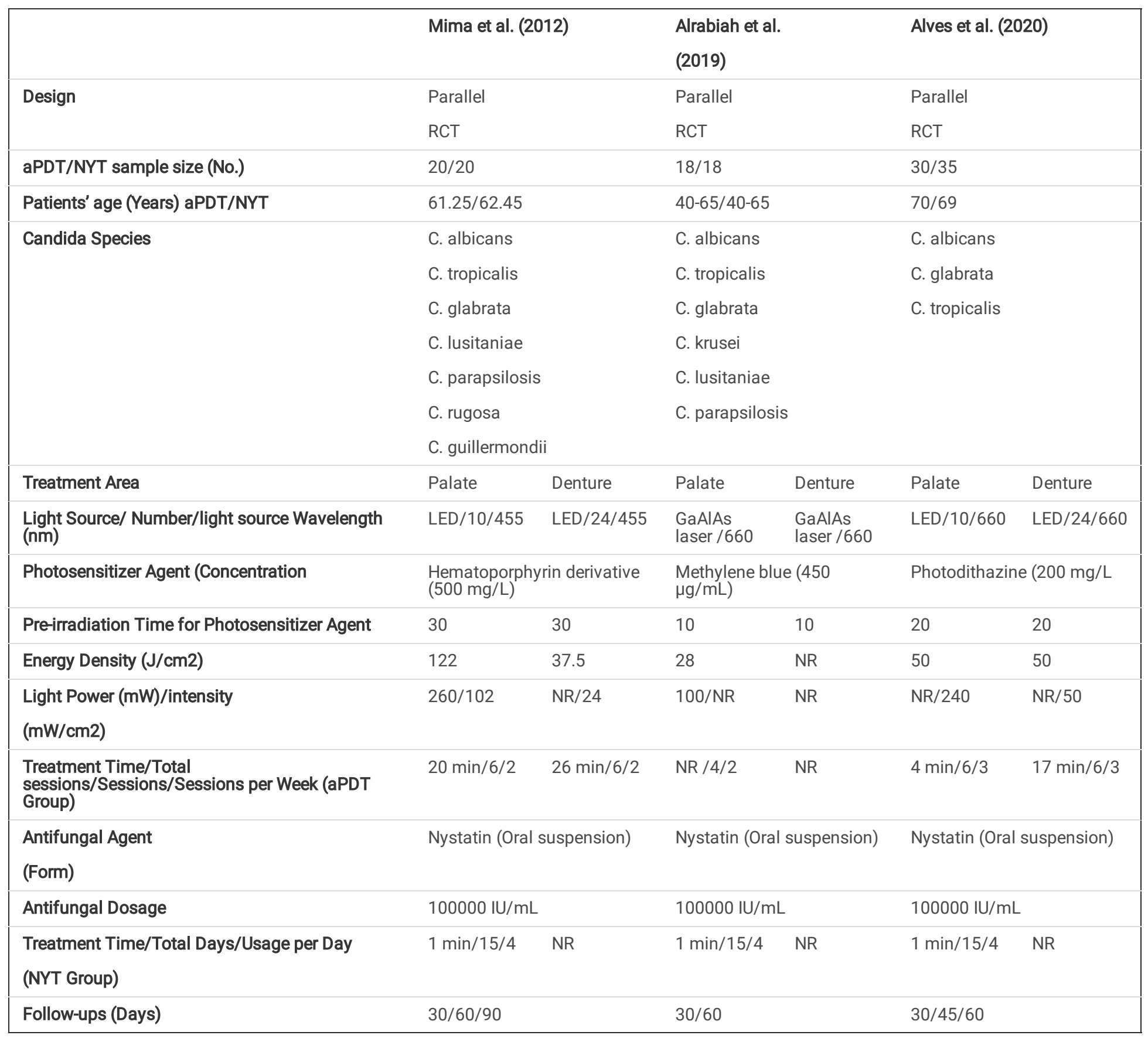

aPDT: Antimicrobial Photodynamic Therapy; NYT: Nystatin Therapy; LED: Light Emitting Diode; NA: Not Reported

Table 2 Summary of evidence.

\begin{tabular}{|c|c|c|c|c|c|c|c|c|c|c|}
\hline \multicolumn{7}{|c|}{ Certainty assessment } & \multicolumn{2}{|c|}{$\begin{array}{l}\text { No of } \\
\text { patients }\end{array}$} & \multirow[t]{2}{*}{ Certainty } & \multirow[t]{2}{*}{ Importance } \\
\hline $\begin{array}{l}\text { № of } \\
\text { studies }\end{array}$ & $\begin{array}{l}\text { Study } \\
\text { design }\end{array}$ & $\begin{array}{l}\text { Risk of } \\
\text { bias }\end{array}$ & Inconsistency & Indirectness & Imprecision & $\begin{array}{l}\text { Other } \\
\text { considerations }\end{array}$ & aPDT & NYS & & \\
\hline \multicolumn{11}{|c|}{ aPDT versus NYT in reducing Candida colony count in patients' palate } \\
\hline 3 & $\begin{array}{l}\text { randomized } \\
\text { trials }\end{array}$ & $\begin{array}{l}\text { serious } \\
\text { a }\end{array}$ & not serious & not serious & serious $^{b}$ & none & 68 & 73 & $\underset{\text { LOW }}{\oplus}$ & CRITICAL \\
\hline \multicolumn{11}{|c|}{ aPDT versus NYT in reducing Candida colony count in patients' denture } \\
\hline 3 & $\begin{array}{l}\text { randomized } \\
\text { trials }\end{array}$ & $\begin{array}{l}\text { serious } \\
\text { a }\end{array}$ & not serious & not serious & serious $^{b}$ & none & 68 & 73 & $\bigoplus_{\text {LOW }}^{\oplus 0}$ & CRITICAL \\
\hline
\end{tabular}




\section{Figures}

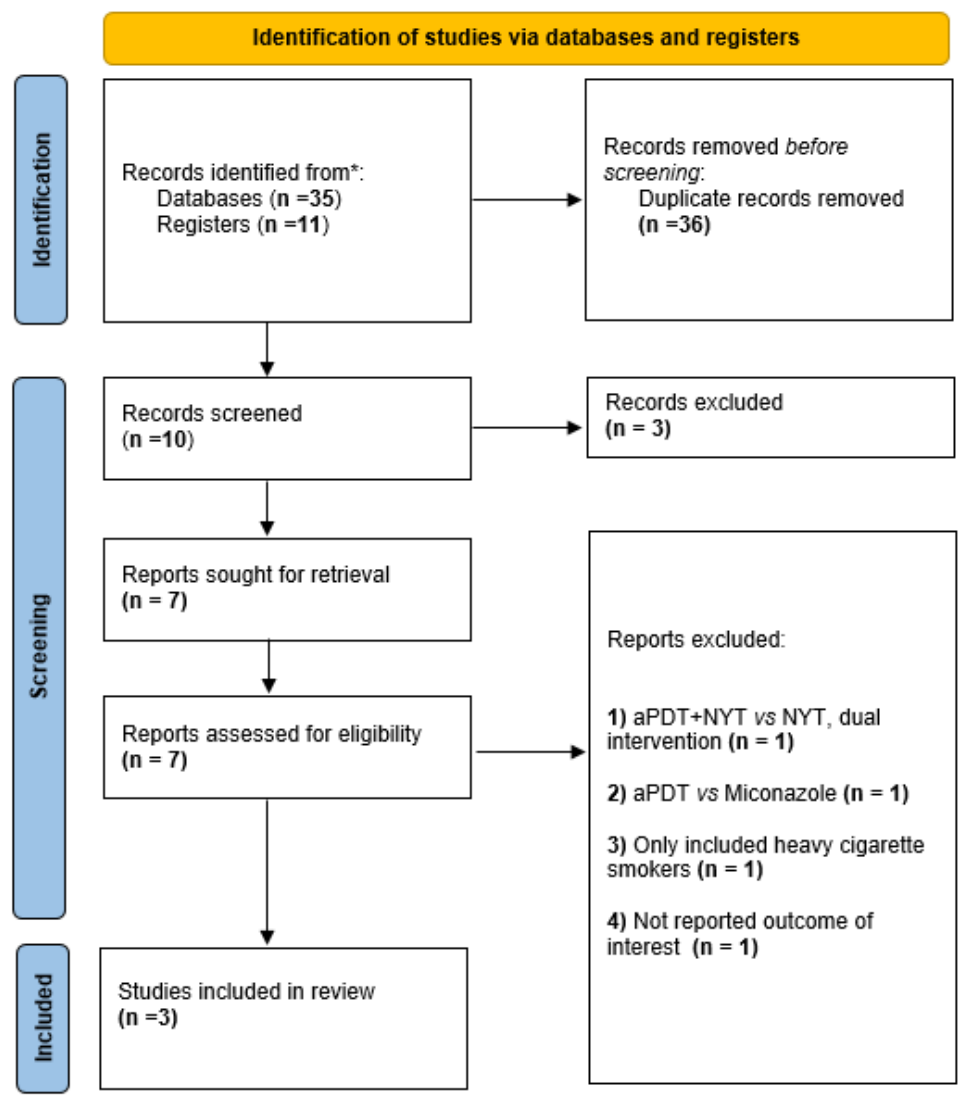

Figure 1

PRISMA flow diagram showing retrieval of papers.

\section{Study ID}

Mima 2012

Alrabiah 2019

Alves 2020
D1 $\underline{\text { D }}$

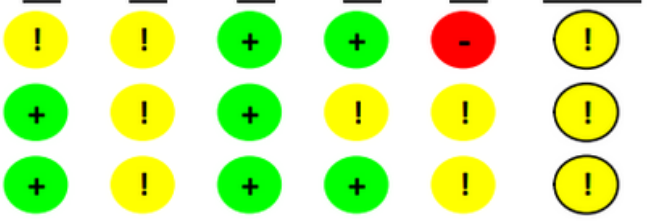

$+\quad$ Low risk

! Some concerns

High risk

\section{D1 Randomisation process \\ D2 Deviations from the intended interventions \\ D3 Missing outcome data \\ D4 Measurement of the outcome \\ D5 Selection of the reported result}

\section{Figure 2}

Risk of bias summary. 


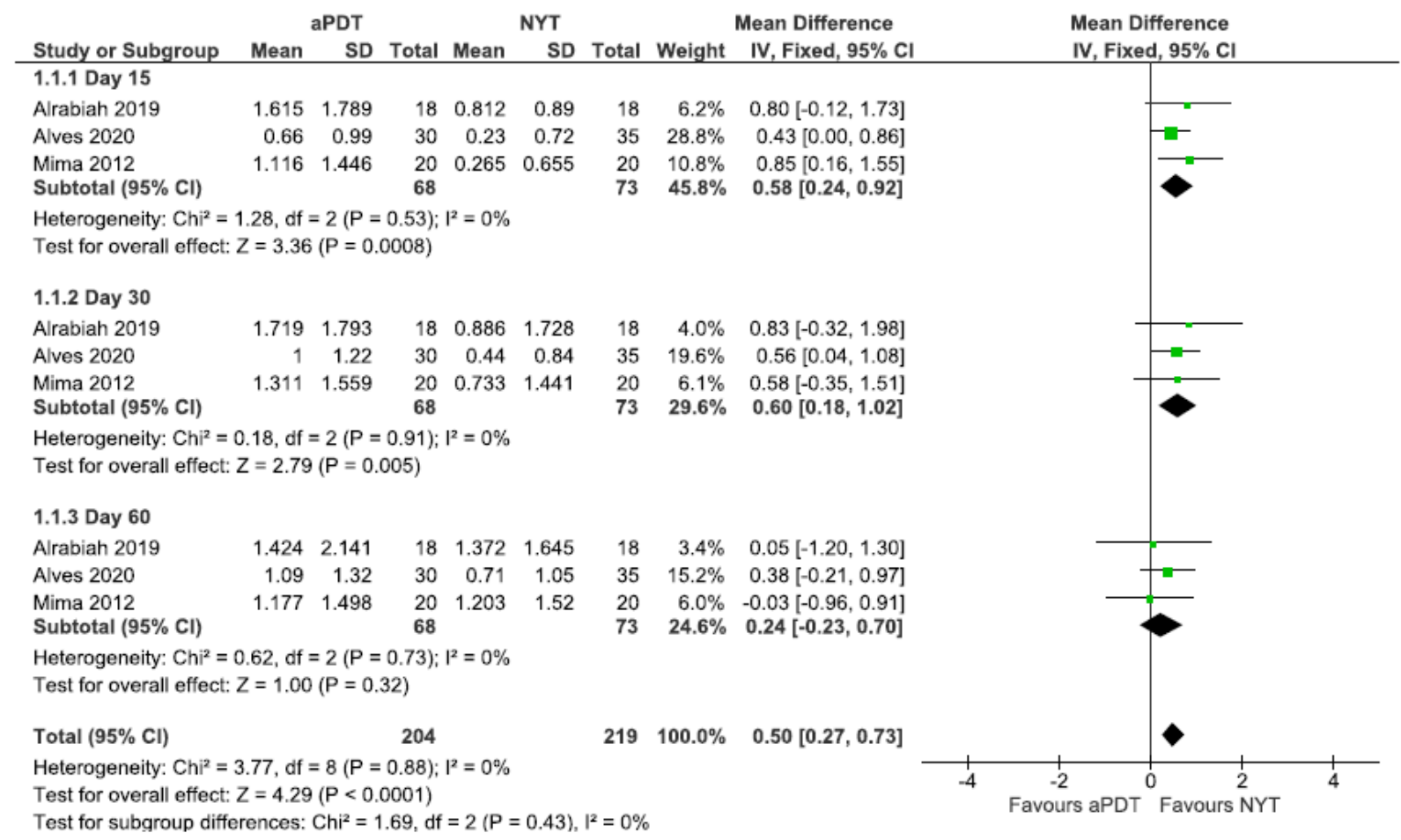

\section{Figure 3}

Forest plot of the mycological efficacy of aPDT compared to NYT in reducing Candida colony count (Log10 CFU/mL) in patients' palate.

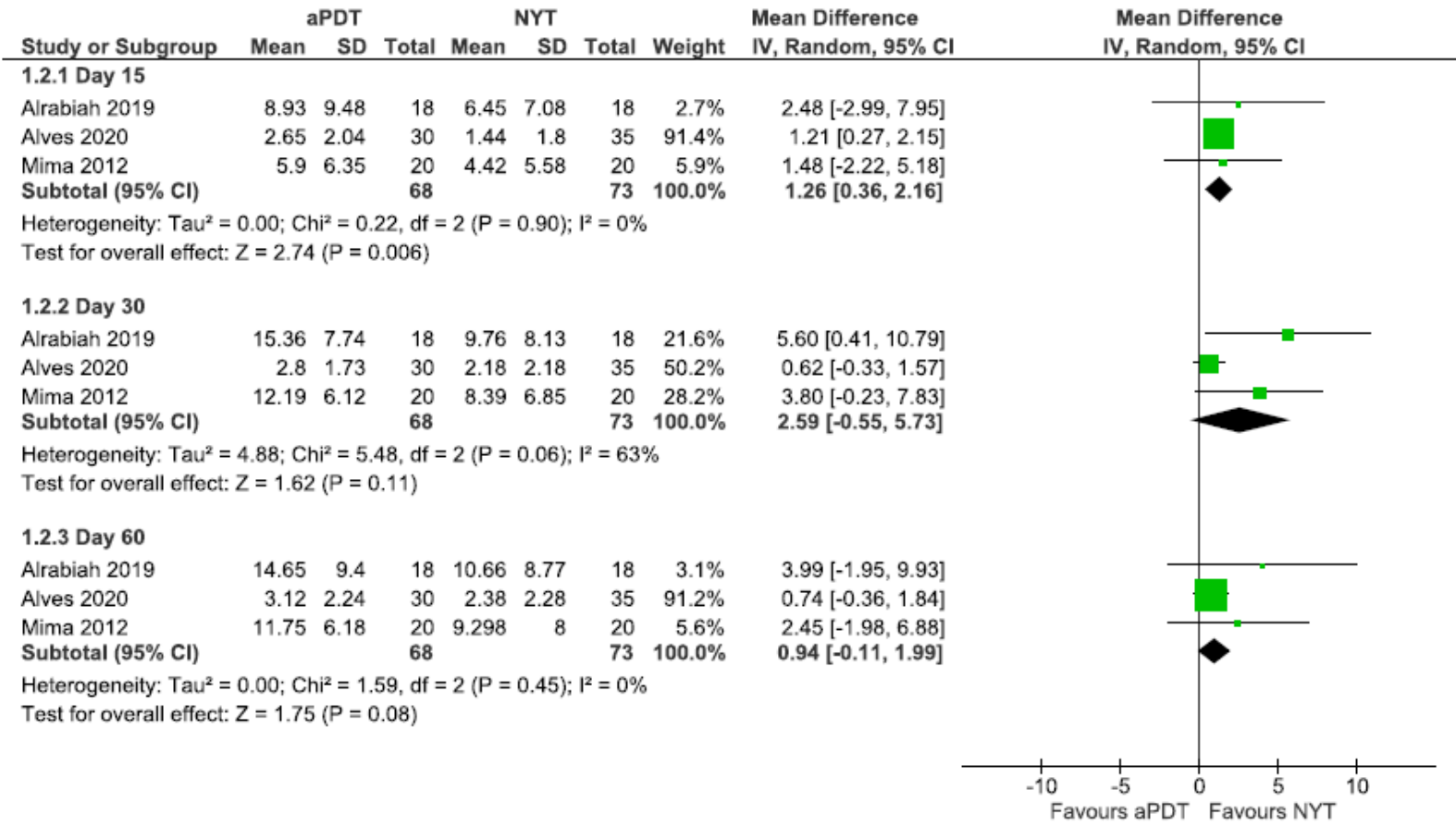

\section{Figure 4}

Forest plot of the mycological efficacy of aPDT compared to NYT in reducing Candida colony count (Log10 CFU/mL) in patients' denture.

\section{Supplementary Files}

This is a list of supplementary files associated with this preprint. Click to download.

- Supplementaryfile1.docx 